\title{
Maladies chroniques et facteurs de risque chez les membres des Premières Nations, les Inuits et les Métis du Nord canadien
}

\author{
S. G. Bruce, Ph. D.; N. D. Riediger, M. Sc.; L. M. Lix, Ph. D.
}

Cet article a fait l'objet d'une évaluation par les pairs.

Diffuser cet article sur Twitter

\section{Résumé}

Introduction : Les populations autochtones du Nord canadien subissent des changements rapides dans leur environnement, ce qui peut avoir des effets nuisibles sur leur état de santé. Nous avons voulu comparer les maladies chroniques et les facteurs de risque des populations autochtones du Nord canadien, à savoir les Premières nations, les Inuits et les Métis, avec les populations non autochtones de la même zone.

Méthodologie : Les données sont tirées de l’Enquête sur la santé dans les collectivités canadiennes de 2005 à 2008. Des modèles de régression logistique multiple pondérée ont servi à analyser l'association entre les groupes ethniques et les résultats de santé. Les covariables du modèle étaient l'âge, le sexe, le territoire de résidence, le niveau de scolarité et le revenu. Nous présentons les rapports de cotes (RC) et nous avons utilisé la méthode d'échantillonnage bootstrap pour calculer les intervalles de confiance (IC) à $95 \%$ et les valeurs $p$.

Résultats : La probabilité d'avoir au moins une maladie chronique était significativement plus faible chez les Inuits ( $\mathrm{RC}=0,59$; IC à $95 \%: 0,43$ à 0,81$)$ que chez les non-Autochtones, mais elle était similaire chez les Premières nations, les Métis et les non-Autochtones. La prévalence de nombreux facteurs de risque était significativement différente chez les Inuits, les membres des Premières nations et les Métis.

Conclusion : Les Autochtones du Nord canadien ont des états de santé hétérogènes. Le maintien d'une surveillance continue des maladies chroniques et des facteurs de risque va jouer un rôle important dans la mesure des évolutions et dans l'évaluation de l'impact des interventions en santé publique les concernant.

Mots-clés : Autochtone, Premières nations, Inuit, Métis, maladie chronique, Nord canadien

\section{Introduction}

La population autochtone du Nord canadien est constituée de trois groupes, les membres des Premières nations, les Inuits et les Métis, avec chacun leur histoire, leur mode de vie et leurs relations avec le gouvernement du Canada. Ce sont les territoires du Nord canadien, c'est-à-dire le Yukon, les Territoires du Nord-Ouest
(T.N.-O.) et le Nunavut, qui comptent la proportion d'Autochtones la plus forte au Canada. En moyenne, 40 \% des Canadiens vivant dans ces territoires sont autochtones, alors que les Autochtones forment seulement $4 \%$ de l'ensemble de la population canadienne ${ }^{1}$. Au Nunavut, $85 \%$ de la population est autochtone, et cette dernière est à plus de $90 \%$ inuite. Dans les T.N.-O., $50 \%$ de la population est autochtone (Premières nations, 61 \%; Inuits, $20 \%$; Métis, $17 \%$ ) et, au Yukon, $25 \%$ de la population est autochtone (Premières nations, $83 \%$; Métis, $11 \%$; Inuit, $4 \%)^{*}$.

Au cours du dernier demi-siècle, la population autochtone du Nord canadien a connu une transition sanitaire importante, caractérisée par une réduction des maladies infectieuses et une augmentation des maladies chroniques, en particulier du diabète, de l'obésité, des maladies cardiaques et des maladies respiratoires. Cette transition s'est accompagnée d'une hausse des problèmes de société, en particulier de la violence, des accidents et de la toxicomanie $^{2}$. Ces phénomènes sont étroitement reliés et sont attribuables aux effets de la colonisation et aux changements subséquents qu'ont subi les milieux autant physiques que sociaux ${ }^{3}$.

La transition épidémiologique se déroule à une vitesse différente chez les Autochtones du Sud et ceux du Nord canadien. Comme Lix et ses collègues ${ }^{4}$ l'ont montré, les maladies chroniques et leurs facteurs de risque représentent un lourd fardeau dans le Sud et un fardeau qui s'accroît dans le Nord. La surveillance des maladies chroniques et des facteurs de risque a un rôle important à jouer au sein des populations dont l'état de santé se modifie rapidement car elle peut contribuer à l'élaboration des interventions. Il est en effet important, pour les organismes dirigeants des Premières nations, des Inuits et des Métis, de comprendre les enjeux auxquels sont confrontés ces populations et d'agir en fonction du caractère spécifique de chacun de ces groupes autochtones et des relations parti-

* La somme des pourcentages n'est pas de $100 \%$, car les personnes s'identifiant à plus d'un groupe ethnique ont été exclues.

Rattachement des auteurs :

Département des sciences de la santé communautaire, Faculté de médecine, Université du Manitoba, Winnipeg (Manitoba), Canada

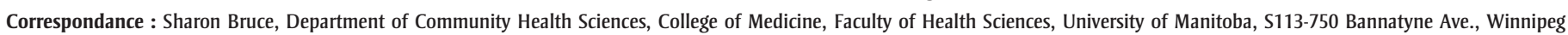
(Manitoba) R3E 0W3; tél. : 204-975-7745; téléc. : 204-789-3905; courriel : sharon.bruce@med.umanitoba.ca 
culières que chacun entretient avec les gouvernements fédéral, provinciaux et locaux. Pour cela, il faut disposer pour chaque groupe autochtone de données qui lui soient spécifiques, quel que soit le ressort territorial.

Peu de travaux ont comparé les prévalences des maladies chroniques et de leurs facteurs de risque et de protection au sein des trois populations autochtones du Nord canadien. Les objectifs de cette recherche étaient de décrire et de comparer la prévalence des maladies chroniques et de leurs facteurs de risque chez les Premières nations, les Inuits et les Métis et de comparer ces populations aux populations non autochtones du Nord.

\section{Méthodologie}

\section{Source des données}

Pour cette recherche, nous avons utilisé les données des cycles 3.1 (2005-2006) et 4.1 (2007-2008) de l'Enquête sur la santé dans les collectivités canadiennes (ESCC). L'ESCC est une enquête menée par Statistique Canada à l'échelle du pays à l'aide de questions sur l'état de santé, sur les déterminants de la santé et sur l'utilisation du système de soins de santé dans 136 régions sanitaires du Canada. Cette enquête couvre environ $98 \%$ de la population canadienne de 12 ans et plus. Les habitants des réserves indiennes ou d'autres terres publiques, les personnes vivant en établissement et les membres à temps plein des Forces canadiennes sont exclus de cette enquête. Au Yukon et au Nunavut, les Autochtones ne vivent pas dans des réserves, et il en est de même pour plus de $99 \%$ de la population des T.N.-O. ${ }^{5}$. Au Nunavut, l'ESCC n'a recueilli des données que dans les dix plus grandes collectivités, mais cela représente néanmoins $71 \%$ de la population de ce territoire $^{6}$. Nous avons combiné les données des deux cycles de l'ESCC pour obtenir un échantillonnage suffisant pour l'étude de diverses maladies chroniques et facteurs de risque.

La taille des échantillons était de 132947 personnes pour le cycle 3.1 de l'ESCC et de 131959 personnes pour le cycle 4.1. Pour le cycle 3.1, le taux de réponse était de
78,9 \% pour l'ensemble du Canada, de $81,6 \%$ pour le Yukon, de $81,7 \%$ pour les T.N.-O. et de $87,7 \%$ pour le Nunavut. Pour le cycle 4.1, le taux de réponse était de $76,4 \%$ pour l'ensemble du Canada, de $83,0 \%$ pour le Yukon, de 85,0 \% pour les T.N.-O. et de $85,4 \%$ pour le Nunavut. Notre étude a porté sur tous les répondants du cycle 3.1 et du cycle 4.1 âgés de 20 ans et plus ayant déclaré comme région de résidence le Yukon, les T.N.-O. ou le Nunavut. Cela signifie que notre population non autochtone de référence réside aussi dans le Nord canadien.

Cette recherche a été approuvée par le conseil d'éthique de la recherche en santé de l’Université du Manitoba. Statistique Canada a autorisé l'accès aux données. Les analyses ont été menées dans un environnement sécurisé, au Centre de données de recherche de Statistique Canada de l'Université du Manitoba.

\section{Mesures}

Dans chaque cycle de l'ESCC, on demandait aux répondants s'ils s'identifiaient à l'un des trois groupes autochtones reconnus par la Constitution. Les répondants qui s'identifiaient à plus d'un de ces groupes ont été considérés comme faisant partie des Premières nations. Étant donné la petite taille de l'échantillon, nous ne souhaitions exclure personne. Les répondants ayant choisi un groupe ethnique autre que l'un de ces trois groupes autochtones ont été considérés comme non autochtones. Pour cette étude, les catégories ethniques sont donc les suivantes : Premières nations, Inuits, Métis et non-Autochtones.

Les caractéristiques associées aux répondants étaient, outre l'âge et le sexe, le niveau de scolarité et le revenu total du ménage. Le niveau de scolarité a été réparti en trois catégories : études secondaires non terminées, diplôme d'études secondaires et études postsecondaires. Les répondants devaient fournir une estimation du revenu total du ménage au cours des 12 mois précédant l'enquête incluant toutes leurs sources de revenu avant impôt et déductions fiscales. Nous avons créé quatre catégories pour cette variable : 0 à 29999 \$, 30000 \$ à 59999 \$, 60000 \$ à 99999 \$ et 100000 \$ et plus.
On demandait également aux répondants de quelles affections chroniques durant en général 6 mois ou plus (ou déjà présentes depuis au moins ce laps de temps) et diagnostiquées par un professionnel de la santé ils souffraient. Notre analyse a porté sur un nombre important de maladies chroniques : arthrite ou rhumatisme, asthme, maladies de l'intestin, cancer, diabète, emphysème ou maladie pulmonaire obstructive chronique (MPOC), maladie cardiaque, hypertension et accident vasculaire cérébral (AVC). Des variables dichotomiques (présence/absence) ont été créées pour chacune. De plus, une variable spécifique a été créée pour caractériser la morbidité globale de chaque individu. Il s'agit d'une variable binaire de la morbidité créée sur la base de la présence ou non d'au moins une des maladies chroniques suivantes : arthrite ou rhumatisme, asthme, hypertension, diabète, maladie cardiaque, cancer, AVC, bronchite chronique, emphysème, MPOC, maladie de l'intestin, troubles anxieux, troubles de l'humeur, migraines, démence, ulcères gastriques ou intestinaux, incontinence urinaire et maux de dos.

Nous avons aussi examiné un certain nombre de facteurs de risque et de protection : la consommation d'alcool, le tabagisme, l'indice de masse corporelle (IMC), l'activité physique durant les loisirs et l'activité physique régulière. Nous avons exclu les facteurs de risque faisant partie du module facultatif de l'ESCC. Nous avons catégorisé la consommation d'alcool comme suit : non-consommation, consommation occasionnelle (moins d'1 verre par mois dans les 12 derniers mois), consommation régulière (1 verre ou plus par mois dans les 12 derniers mois) et consommation abusive (5 verres ou plus à au moins une occasion dans les 12 derniers mois) ${ }^{7}$. Les réponses possibles liées à la fréquence du tabagisme (cigarettes) étaient les suivantes : tabagisme quotidien, tabagisme occasionnel et nonfumeur. Des variables dichotomiques (oui/non) ont été créées pour chacune des catégories. Par exemple, le rapport de cotes du tabagisme quotidien a été comparé au rapport de cotes des non-fumeurs. Nous avons créé des variables dichotomiques pour faciliter l'interprétation et réduire au maximum l'effet lié aux faibles effectifs dans certaines catégories au 
moment du recoupement des données avec les variables explicatives. Le choix des maladies chroniques et des facteurs de risque repose à la fois sur leur présence dans l'ensemble de données et sur des considérations théoriques : un grand nombre de maladies chroniques jouent déjà un rôle dans la transition épidémiologique du Nord ${ }^{4,8}$. Tous ces facteurs de risque ont déjà été corrélés à plusieurs maladies chroniques, sont interdépendants ou sont des marqueurs de facteurs structurels et liés plus largement aux collectivités. Par exemple, la consommation d'alcool est associée aux maladies cardiaques, à l'hypertension, aux troubles anxieux, aux troubles de l'humeur et aux maladies de l'intestin 9-11 . Le tabagisme est associé à l'asthme, à la bronchite chronique, au diabète, aux maladies cardiaques et à l'hypertension ${ }^{12}$. L'embonpoint et l'obésité sont associés à l'arthrite, à l'asthme, au diabète, aux maladies cardiaques, à l'hypertension, aux maladies de l'intestin, aux troubles anxieux et aux troubles de l'humeur ${ }^{13,14}$.

L'IMC a été calculé à l'aide de données autodéclarées sur la taille et le poids ${ }^{15}$. L'embonpoint correspond à un IMC situé entre 25,00 et $29,99 \mathrm{~kg} / \mathrm{m}^{2}$ et l'obésité à un IMC de $30,0 \mathrm{~kg} / \mathrm{m}^{2}$ et plus ${ }^{16}$.

Les répondants devaient déclarer la fréquence de leurs activités physiques non professionnelles d'une durée de plus de 15 minutes effectuées dans les 3 mois précédant la date de l'entrevue. Une fréquence moyenne mensuelle a été ensuite calculée. L'activité physique a été catégorisée comme suit : régulière (12 fois par mois ou plus); occasionnelle (4 à 11 fois par mois) et rare (moins de 4 fois par mois). Des variables dichotomiques (oui/non) ont été créées pour chaque catégorie d'activité physique. Le degré d'activité physique durant les loisirs a été calculé en fonction de la dépense énergétique totale quotidienne pendant ces activités ${ }^{17}$ et nous avons classé chaque répondant comme actif (3,0 kcal ou plus par kg et par jour), modéré (1,5 à 2,99 kcal par kg et par jour) ou inactif (0 à 1,49 kcal par $\mathrm{kg}$ et par jour). Les activités physiques réalisées durant les loisirs étaient entre autres la marche, la course, le cyclisme, la natation, les exercices à la maison, les classes d'exercices ou aérobie, la pêche et le jardinage, ainsi que la pratique de sports d'équipe comme le hockey, le basketball, le volley-ball ou le soccer. Des variables dichotomiques (oui/non) ont été créées pour chacune des catégories d'activité physique durant les loisirs.

Nous avons analysé également la mesure globale de l'état de santé. Les répondants devaient évaluer leur état de santé selon une échelle à 5 choix variant d'excellent à mauvais. Nous avons réparti ces 5 choix de réponse en 2 catégories : excellent, très bon et bon dans une catégorie, et correct et mauvais dans l'autre (voir Bombak et Bruce $^{18}$ pour davantage de renseignements sur l'autoévaluation de l'état de santé des populations autochtones).

\section{Analyse des données}

Les données des deux cycles ont été combinées à l'aide de la méthode des estimations groupées ${ }^{19}$. Les analyses descriptives portant sur le nombre total de répondants et leurs caractéristiques sociodémographiques ont été menées avec des données non pondérées. Comme la prévalence brute des maladies chroniques, des facteurs de risque et des états de santé autoévalués a été calculée avec des intervalles de confiance (IC) à $95 \%$ et des poids d'échantillonnage, les estimations sont représentatives de la population à l'étude.

Des analyses de régression logistique multiple pondérée ont servi à qualifier la relation entre l'ethnicité et chaque mesure des maladies chroniques, des facteurs de risque pour la santé et de l'état de santé autoévalué. Outre le groupe ethnique, les covariables étaient le groupe d'âge (20 à 34 ans, 35 à 54 ans, 55 ans et plus), le sexe, le territoire de résidence, le niveau de scolarité et le revenu total du ménage. Les catégories de référence étaient le groupe des 55 ans et plus, les hommes, l'ethnicité non autochtone dans le cas des analyses portant sur tous les groupes ethniques et les Premières nations dans le cas des analyses portant sur les populations autochtones, les T.N.-O. comme lieu de résidence, les études secondaires non terminées et le revenu le moins élevé (0 \$ à 29999 \$).
Nous avons utilisé la méthode d'échantillonage bootstrap pour calculer les IC à $95 \%$ des estimations de la prévalence brute et des rapports de cotes corrigés (RCC ${ }^{20,21}$. Cette méthode permet de procéder à un échantillonnage aléatoire avec remplacement de l'ensemble initial des observations et de produire une distribution d'échantillonnage pour un paramètre de population donné. Nous avons réalisé toutes les analyses à l'aide de la macro du logiciel $\mathrm{SAS}^{22}$ mise au point par des spécialistes de la méthodologie de Statistique Canada. Cette analyse a porté sur 500 échantillons, conformément aux recommandations des développeurs du logiciel.

\section{Résultats}

Dans le tableau 1 sont présentées les caractéristiques sociodémographiques de la population à l'étude. Les données manquantes étaient rares (moins de $1 \%$ ). Les membres des Premières nations et les Inuits étaient plus jeunes que les nonAutochtones : $59 \%$ des membres des Premières nations et $74 \%$ des Inuits étaient âgés de moins de 45 ans, contre $50 \%$ des non-Autochtones. La structure par âge des Métis était semblable à celle des nonAutochtones. Le niveau de scolarité était plus faible chez Autochtones que chez les non-Autochtones. Le revenu annuel était également plus faible chez les membres des Premières nations et les Inuits que chez les Métis et les non-Autochtones.

La prévalence brute des maladies chroniques et des facteurs de risque est présentée dans le tableau 2 et les RCC dans le tableau 3. La population non autochtone est le groupe de référence dans les analyses de régression. Le RCC de la présence d'au moins une maladie chronique était significativement plus faible chez les Inuits que dans la population non autochtone, mais similaire chez les membres des Premières nations, les Métis et les populations non autochtones. Les maladies chroniques les plus fréquentes, toutes populations confondues, étaient l'arthrite et l'hypertension. Le RCC du diabète était significativement plus faible chez les Inuits que chez les non-Autochtones. D'autres maladies chroniques comme l'asthme, les maladies de l'intestin (p. ex. maladie de Crohn, colite ulcéreuse ou 
TABLEAU 1

Caractéristiques sociodémographiques de la population du Nord canadien âgée de 20 ans et plus en 2005 et en 2008

\begin{tabular}{|c|c|c|c|c|c|c|c|c|c|}
\hline \multicolumn{2}{|c|}{ Caractéristiques } & \multicolumn{2}{|c|}{ Premières Nations } & \multicolumn{2}{|c|}{ Métis } & \multicolumn{2}{|c|}{ Inuits } & \multicolumn{2}{|c|}{ Non-Autochtones } \\
\hline & & $\mathbf{n}$ & $\%$ & n & $\%$ & $\mathbf{n}$ & $\%$ & $\mathrm{n}$ & $\%$ \\
\hline Sexe & Homme & 363 & 43,9 & 97 & 44,9 & 452 & 48,5 & 1486 & 49,6 \\
\hline \multirow[t]{2}{*}{ Groupe d'âge (ans) } & 20 à 34 & 282 & 33,9 & 67 & 31,0 & 444 & 47,6 & 851 & 28,4 \\
\hline & 35 à 44 & 204 & 24,6 & 58 & 26,9 & 245 & 26,3 & 649 & 21,7 \\
\hline \multirow[t]{3}{*}{ Niveau de scolarité } & Études secondaires non terminées & 374 & 45,0 & 49 & 22,7 & 428 & 45,9 & 281 & 9,4 \\
\hline & Diplôme d'études secondaires & 74 & 8,9 & 26 & 12,0 & 68 & 7,3 & 325 & 10,8 \\
\hline & Études postsecondaires & 377 & 45,4 & 141 & 65,3 & 429 & 46,0 & 2379 & 79,4 \\
\hline Revenu total du ménage (\$) & 0 à 29999 & 277 & 33,3 & 32 & 14,8 & 304 & 32,6 & 345 & 11,5 \\
\hline Échantillon total (N) & & 831 & 16,6 & 216 & 4,3 & 932 & 18,6 & 2997 & 59,8 \\
\hline
\end{tabular}

TABLEAU 2

Prévalence brute (\%) des maladies chroniques et des facteurs de risque et de protection selon le groupe ethnique, 2005 et 2008

\begin{tabular}{|c|c|c|c|c|}
\hline & \multicolumn{4}{|c|}{ Prévalence, en \% (IC à 95 \%) } \\
\hline & Premières nations & Métis & Inuits & Non-Autochtones \\
\hline \multicolumn{5}{|l|}{ Maladie chronique } \\
\hline Au moins 1 maladie chronique & $51,88(46,83-56,93)$ & $52,66(43,36-61,96)$ & $34,64(29,96-39,33)$ & $50,60(47,58-53,61)$ \\
\hline Arthrite & $12,43(9,46-15,39)$ & $14,03(8,92-19,14)$ & $11,02(8,11-13,92)$ & $13,98(12,44-15,52)$ \\
\hline Asthme & $6,43(3,86-8,99)$ & $7,90(4,09-11,71)$ & $3,51(1,78-5,23)$ & $8,38(7,09-9,67)$ \\
\hline Bronchite chronique & $2,06(1,03-3,10)$ & $3,07(0,00-6,39)$ & $0,92(0,16-1,68)$ & $1,28(0,71-1,85)$ \\
\hline Diabète & $5,22(3,51-6,93)$ & $5,67(1,13-10,20)$ & $1,02(0,41-1,62)$ & $4,10(3,21-4,99)$ \\
\hline Maladie cardiaque & $3,31(1,69-4,92)$ & $3,94(0,75-7,14)$ & $2,14(0,85-3,42)$ & $2,73(1,97-3,50)$ \\
\hline Hypertension & $13,45(10,32-16,58)$ & $12,88(7,16-18,61)$ & $7,76(5,97-9,56)$ & $12,82(11,26-14,39)$ \\
\hline Trouble anxieux & $3,66(2,26-5,06)$ & $6,58(3,43-9,74)$ & $2,63(0,69-4,57)$ & $3,65(2,68-4,62)$ \\
\hline Maladie de l'intestin & $3,43(1,47-5,39)$ & $4,55(0,81-8,28)$ & $0,80(0,00-1,70)$ & $4,18(3,13-5,23)$ \\
\hline Trouble de l'humeur & $5,46(3,74-7,19)$ & $5,62(1,56-9,69)$ & $3,05(1,56-4,54)$ & $6,88(5,42-8,34)$ \\
\hline \multicolumn{5}{|l|}{ Facteur de risque ou de protection } \\
\hline Consommation abusive d'alcool ${ }^{\mathrm{a}}$ & $72,83(68,19-77,47)$ & $64,81(55,07-74,55)$ & $68,82(64,73-72,90)$ & $54,45(51,42-57,48)$ \\
\hline Consommation régulière d'alcool ${ }^{\text {b }}$ & $54,84(49,14-60,53)$ & $67,95(60,54-75,36)$ & $45,15(37,47-52,84)$ & $67,48(64,42-70,55)$ \\
\hline Tabagisme quotidien & $45,13(40,97-49,28)$ & $33,07(24,77-41,37)$ & $63,62(59,21-68,03)$ & $22,84(20,33-25,35)$ \\
\hline Embonpoint $^{c}$ & $29,04(25,58-32,51)$ & $31,52(22,19-40,85)$ & $26,75(23,48-30,01)$ & $33,52(31,25-35,79)$ \\
\hline Obésitéd & $23,55(18,54-28,56)$ & $28,28(20,43-36,12)$ & $24,27(20,48-28,06)$ & $21,05(19,06-23,03)$ \\
\hline Activité physique durant les loisirs ${ }^{\mathrm{e}}$ & $19,37(14,54-24,21)$ & $18,83(12,94-24,73)$ & $18,48(14,73-22,24)$ & $23,34(20,77-25,90)$ \\
\hline Activité physique régulière ${ }^{f}$ & $55,38(49,30-61,47)$ & $57,08(49,15-65,00)$ & $47,52(42,57-52,47)$ & $65,35(62,72-67,98)$ \\
\hline État de santé autoévaluégg & $82,64(79,49-85,79)$ & $88,51(82,20-94,82)$ & $82,98(79,83-86,13)$ & $91,05(89,42-92,68)$ \\
\hline
\end{tabular}

Abréviations : IC, intervalle de confiance; IMC, indice de masse corporelle.

a 5 verres ou plus à au moins une occasion au cours des 12 derniers mois.

b 1 verre ou plus par mois au cours des 12 derniers mois.

c IMC entre 25,00 et $29,99 \mathrm{~kg} / \mathrm{m}^{2}$.

d IMC supérieur ou égal à $30,0 \mathrm{~kg} / \mathrm{m}^{2}$.

e $3,0 \mathrm{kcal} / \mathrm{kg} / \mathrm{jour}$ ou plus.

f 12 fois ou plus par mois.

g Trois des choix de réponse concernant l'autoévaluation de l'état de santé combinés en un seul : excellent, très bon et bon. 
TABLEAU 3

Rapports de cotes corrigés des principales maladies chroniques et des facteurs de risque et de protection selon le groupe ethnique, 2005 et 2008

\begin{tabular}{|c|c|c|c|}
\hline & \multicolumn{3}{|c|}{ RRC (IC à $95 \%$ ) } \\
\hline & Premières nations & Métis & Inuits \\
\hline \multicolumn{4}{|l|}{ Maladie chronique } \\
\hline Au moins 1 maladie chronique & $0,95(0,72-1,27)$ & $1,09(0,72-1,66)$ & $0,59(0,43-0,81)^{\mathrm{a}}$ \\
\hline Arthrite & $0,70(0,48-1,03)$ & $1,06(0,67-1,68)$ & $0,90(0,56-1,46)$ \\
\hline Asthme & $0,69(0,42-1,13)$ & $0,92(0,50-1,68)$ & $0,48(0,24-0,96)^{\mathrm{a}}$ \\
\hline Bronchite chronique & $1,00(0,41-2,49)$ & $2,24(0,20-24,74)$ & $0,68(0,11-4,40)$ \\
\hline Diabète & $1,26(0,75-2,13)$ & $1,58(0,64-3,90)$ & $0,36(0,14-0,88)^{\mathrm{a}}$ \\
\hline Maladie cardiaque & $0,91(0,48-1,72)$ & $1,81(0,55-5,92)$ & $1,06(0,45-2,49)$ \\
\hline Hypertension & $1,12(0,74-1,69)$ & $1,22(0,68-2,21)$ & $0,77(0,51-1,17)$ \\
\hline Trouble anxieux & $1,02(0,61-1,68)$ & $2,07(1,07-4,03)^{\mathrm{a}}$ & $0,75(0,32-1,79)$ \\
\hline Maladie de l'intestin & $0,63(0,33-1,22)$ & $1,03(0,41-2,56)$ & $0,15(0,05-0,41)^{\mathrm{a}}$ \\
\hline Trouble de l'humeur & $0,66(0,42-1,03)$ & $0,76(0,32-1,78)$ & $0,37(0,19-0,72)^{\mathrm{a}}$ \\
\hline \multicolumn{4}{|l|}{ Facteur de risque } \\
\hline Consommation abusive d'alcool $^{b}$ & $2,19(1,58-3,04)^{\mathrm{a}}$ & $1,46(0,91-2,34)$ & $1,85(1,23-2,78)^{a}$ \\
\hline Consommation régulière d'alcool ${ }^{\mathrm{C}}$ & $0,77(0,57-1,03)$ & $1,12(0,74-1,68)$ & $0,46(0,31-0,67)^{\mathrm{a}}$ \\
\hline Tabagisme quotidien & $2,09(1,60-2,74)^{a}$ & $1,54(1,07-2,21)^{\mathrm{a}}$ & $3,48(2,43-4,98)^{a}$ \\
\hline Embonpoint $^{\mathrm{d}}$ & $0,94(0,73-1,21)$ & $0,89(0,55-1,44)$ & $0,79(0,58-1,07)$ \\
\hline Obésitée $^{-}$ & $1,36(0,98-1,89)$ & $1,51(1,03-2,19)^{\mathrm{a}}$ & $1,37(0,93-2,03)$ \\
\hline Activité physique durant les loisirs ${ }^{f}$ & $0,96(0,68-1,37)$ & $0,88(0,56-1,39)$ & $0,82(0,59-1,12)$ \\
\hline Activité physique régulière ${ }^{g}$ & $0,93(0,69-1,25)$ & $0,84(0,59-1,18)$ & $0,76(0,55-1,05)$ \\
\hline État de santé auto-évaluéh & $0,72(0,52-0,99)$ & $0,84(0,41-1,71)$ & $0,55(0,34-0,88)^{\mathrm{a}}$ \\
\hline
\end{tabular}

Abréviations : IC, intervalle de confiance; IMC, indice de masse corporelle; RRC, rapport de cotes corrigé.

Remarques : Les rapports de cotes ont été corrigés en fonction de l'âge, du sexe, de la région, du revenu et du niveau de scolarité.

Les non-Autochtones constituent le groupe de référence.

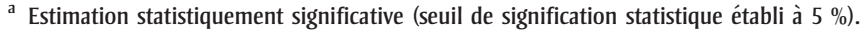

b 5 verres ou plus à au moins une occasion au cours des 12 derniers mois.

c 1 verre ou plus par mois au cours des 12 derniers mois.

d IMC entre 25,00 et $29,99 \mathrm{~kg} / \mathrm{m}^{2}$.

e IMC supérieur ou égal à $30,0 \mathrm{~kg} / \mathrm{m}^{2}$.

f $3,0 \mathrm{kcal} / \mathrm{kg} /$ jour ou plus.

g 12 fois ou plus par mois

h Trois des choix de réponse concernant l'auto-évaluation de l'état de santé combinés en un seul : excellent, très bon et bon.

syndrome du côlon irritable) et les troubles de l'humeur (p. ex. dépression) étaient aussi significativement plus rares chez les Inuits que chez les nonAutochtones. La probabilité de déclarer un trouble anxieux était significativement plus élevée chez les Métis que chez les non-Autochtones. La probabilité de déclarer une maladie chronique n'était pas significativement différente entre les répondants du Nord membres des Premières nations et les non autochtones.

La prévalence de nombreux facteurs de risque des maladies chroniques était les Autochtones que chez les nonAutochtones, avec un maximum de $64 \%$ chez les Inuits et un minimum de $23 \%$ dans la population non autochtone. Le RCC du tabagisme quotidien était 3,5 fois plus élevé chez les Inuits, deux fois plus élevé chez les membres des Premières nations et 1,5 fois plus élevé chez les Métis.

Environ $30 \%$ de tous les répondants présentaient de l'embonpoint, et la prévalence de l'obésité variait entre $24 \%$ et $28 \%$ chez les répondants membres des Premières nations, inuits et métis, contre $21 \%$ chez les répondants non autochtones. Le RCC de l'obésité chez les Métis était équivalent à 1,51 fois celui de la population non autochtone. La proportion de répondants membres des Premières nations, inuits et métis ayant déclaré une activité physique régulière et une activité physique durant leurs loisirs est plus faible que celle des répondants non autochtones. Une forte proportion des répondants ont déclaré que leur état de santé était excellent, très bon ou bon : 83 \% des répondants membres des Premières nations et des répondants inuits et $91 \%$ des répondants non autochtones. Toutefois, le RCC de la déclaration d'un état de santé excellent, très bon ou bon était significativement plus faible chez les Inuits que chez les non-Autochtones.

Les RCC des affections chroniques et des facteurs de risque des trois groupes autochtones sont présentés dans le tableau 4, les membres des Premières nations constituant le groupe de référence. La probabilité de souffrir de diabète et de maladies de l'intestin était significativement plus faible chez les Inuits que chez les membres des Premières nations. Pour ce qui est des facteurs de risque, le RCC de la consommation régulière d'alcool et celui de l'embonpoint étaient significativement plus faibles chez les Inuits que chez les membres des Premières nations. La probabilité de consommation régulière d'alcool était significativement plus élevée chez les Métis que chez les membres des Premières nations. On n'a relevé aucune autre différence entre les groupes autochtones dans la probabilité d'avoir une maladie chronique, de présenter un facteur de risque ou de déclarer un état de santé donné. 
FIGURE 1

Probabilité de présenter certains facteurs de risque et de déclarer un état de santé donné chez les Autochtones du Nord par rapport aux non-Autochtones du Nord

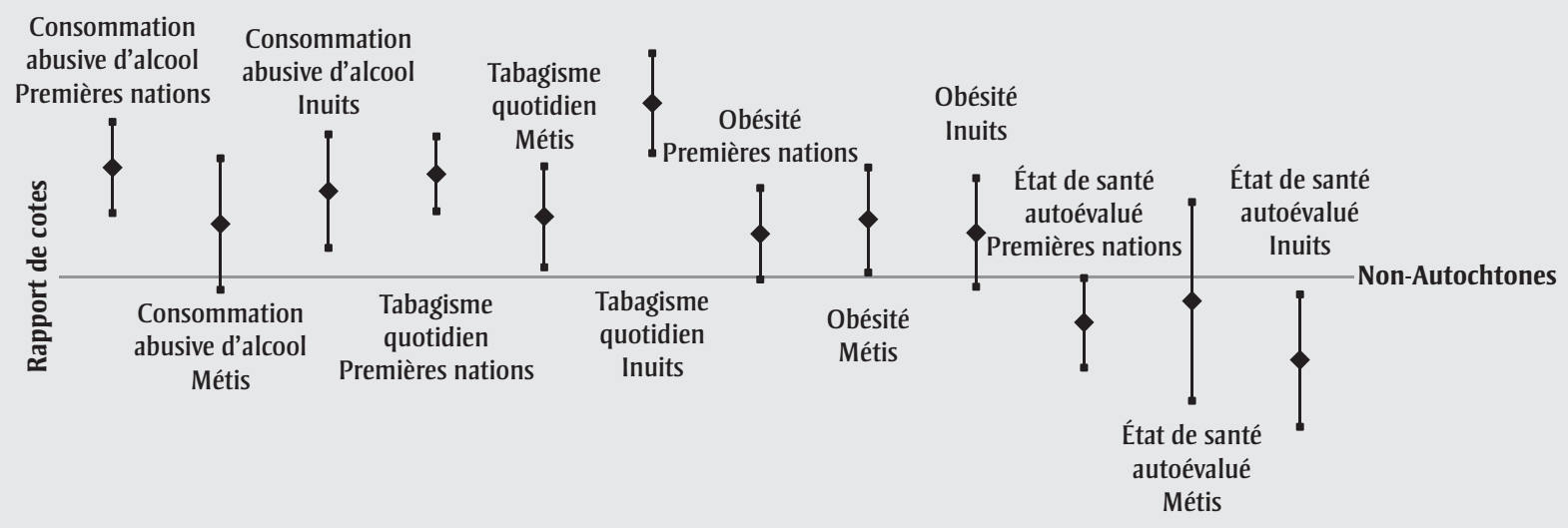

Probabilités de la consommation abusive d'alcool, du tabagisme quotidien, de l'obésité et de l'état de santé autoévalué par groupe ethnique

• Rapport de cotes Limite inférieure de I'IC - Limite supérieure de l'IC

Abréviation : IC, intervalle de confiance.

\section{Analyse}

Nous avons constaté une variabilité de la prévalence des maladies chroniques et des facteurs de risque chez les membres des Premières nations, les Inuits et les Métis du Nord canadien. Dans la plupart des recherches et des rapports de surveillance des maladies chroniques portant sur le Nord canadien, ces trois groupes ethniques ont, jusqu'à maintenant, été combinés en un groupe, celui des Autochtones. Or, ces trois groupes ont chacun leur histoire, leur contexte culturel et leur mode de vie, et ces différences pourraient expliquer la variation observée dans les résultats et avoir une incidence sur les interventions qui leur sont destinées.

Chez les Inuits, la prévalence des maladies chroniques était plus faible que chez les membres des Premières nations et les Métis du Nord. Cela concorde avec les résultats obtenus précédemment avec le diabète ${ }^{23,24}$, les différences interethniques pour d'autres maladies chroniques n'ayant jamais été examinées. Lix et ses collègues ont déjà publié un rapport sur la prévalence des maladies chroniques et des facteurs de risque associés aux Autochtones du Sud (c.-à-d. les résidents des 10 provinces canadiennes) en utilisant les données du cycle 2005-2006 de l'ESCC ${ }^{4}$. Par rapport aux populations autochtones du Sud du Canada, la prévalence de l'arthrite, de l'asthme, des maladies cardiaques, du diabète et de l'hypertension est plus faible chez les Inuits. La prévalence des facteurs de risque des maladies chroniques varie davantage. Les Inuits sont similaires aux membres des Premières nations et aux Métis du Nord en ce qui a trait à la plupart des facteurs de risque étudiés ici. Toutefois, par rapport aux populations autochtones du Sud, la prévalence de l'embonpoint et de la consommation régulière d'alcool est plus faible chez les Inuits, celle de l'obésité est identique et celle de la consommation d'alcool excessive et du tabagisme quotidien sont plus élevées ${ }^{4}$.

Nous avons aussi précédemment mesuré la prévalence des maladies chroniques et des facteurs de risque chez les Autochtones et les non-Autochtones du Nord par territoire de résidence (T.N.-O., Yukon et Nunavut) ${ }^{8}$. Par rapport aux données sur les Autochtones du Nunavut, la prévalence de la plupart des maladies chroniques chez les Inuits n'a pas augmenté, et la prévalence des facteurs de risque est demeurée la même ou a connu une hausse. Ainsi, la prévalence de l'embonpoint et de l'obésité n’a pas changé, mais le tabagisme quotidien, la consommation régulière et la consommation abusive d'alcool ont augmenté ${ }^{8}$.

Le fait que la prévalence des maladies chroniques chez les Inuits soit demeurée la plus faible au sein de toutes les populations autochtones au Canada et n'ait de manière générale pas augmenté depuis 2000 est positif. Cela peut être attribuable à une meilleure adhésion au mode de vie traditionnel de ce groupe, notamment aux habitudes alimentaires. Cependant, la littérature semble aussi indiquer un début d'occidentalisation de l'alimentation ${ }^{25}$. D'un autre côté, l'augmentation de la prévalence des facteurs de risque chez les Inuits est préoccupante. Les facteurs de risque les plus fréquents sont corrélés à des maladies chroniques graves comme le cancer ${ }^{26}$ et les maladies cardiaques $^{27}$ ainsi qu'à des problèmes de société comme la violence, les accidents, les blessures, les dépendances et des dysfonctionnements au sein des familles et des collectivités ${ }^{28}$. Si l'on ajoute à cette constatation le fait que d'autres changements indésirables en lien avec la transition sanitaire aient été rapportés ${ }^{29,30}$, on peut penser que certaines maladies chroniques pourraient être en hausse. Les collectivités, les cliniciens et les décideurs doivent collaborer pour réduire les 
TABLEAU 4

Rapports de cotes corrigés des principales maladies chroniques et des facteurs de risque et de protection selon le groupe ethnique, 2005 et 2008

\begin{tabular}{|c|c|c|}
\hline & \multicolumn{2}{|c|}{ RCC (IC à $95 \%)$} \\
\hline & Métis & Inuits \\
\hline \multicolumn{3}{|l|}{ Maladie chronique } \\
\hline Au moins 1 maladie chronique & $1,19(0,74-1,91)$ & $0,64(0,39-1,06)$ \\
\hline Arthrite & $1,58(0,87-2,88)$ & $1,54(0,73-3,28)$ \\
\hline Asthme & $1,34(0,66-2,70)$ & $1,19(0,44-3,18)$ \\
\hline Bronchite chronique & $2,04(0,18-23,01)$ & $0,67(0,06-7,58)$ \\
\hline Diabète & $1,22(0,53-2,80)$ & $0,33(0,13-0,82)^{\mathrm{a}}$ \\
\hline Maladie cardiaque & $2,22(0,69-7,08)$ & $1,07(0,37-3,10)$ \\
\hline Hypertension & $1,16(0,59-2,26)$ & $0,95(0,51-1,78)$ \\
\hline Trouble anxieux & $2,07(0,98-4,37)$ & $0,60(0,26-1,41)$ \\
\hline Maladie de l'intestin & $1,91(0,64-5,68)$ & $0,27(0,07-0,97)^{\mathrm{a}}$ \\
\hline Trouble de l'humeur & $1,06(0,43-2,60)$ & $0,94(0,30-3,02)$ \\
\hline \multicolumn{3}{|l|}{ Facteur de risque ou de protection } \\
\hline Consommation excessive d'alcool $^{\mathrm{b}}$ & $0,78(0,47-1,30)$ & $1,73(0,91-3,31)$ \\
\hline Consommation régulière d'alcool ${ }^{\mathrm{c}}$ & $1,58(1,09-2,31)^{\mathrm{a}}$ & $0,56(0,32-0,97)^{\mathrm{a}}$ \\
\hline Tabagisme quotidien & $0,69(0,45-1,04)$ & $1,49(0,91-2,45)$ \\
\hline Embonpoint $^{\mathrm{d}}$ & $0,94(0,57-1,54)$ & $0,57(0,36-0,92)^{\mathrm{a}}$ \\
\hline Obésitée $^{\text {On }}$ & $1,03(0,61-1,75)$ & $1,79(0,98-3,27)$ \\
\hline Activité physique durant les loisirs ${ }^{\mathrm{f}}$ & $0,88(0,50-1,56)$ & $0,56(0,29-1,09)$ \\
\hline Activité physique régulière ${ }^{g}$ & $0,96(0,63-1,45)$ & $0,68(0,41-1,13)$ \\
\hline État de santé autoévaluéh & $1,18(0,60-2,33)$ & $0,64(0,36-1,13)$ \\
\hline
\end{tabular}

Abréviations : IC, intervalle de confiance; IMC, indice de masse corporelle; RRC, rapport de cotes corrigé.

Remarques : Les rapports de cotes ont été corrigés en fonction de l'âge, du sexe, de la région, du revenu et du niveau de scolarité.

Les Premières nations constituent le groupe de référence.

${ }^{a}$ Estimation statistiquement significative (seuil de signification statistique établi à $5 \%$ ).

b 5 verres ou plus à au moins une occasion au cours des 12 derniers mois.

c 1 verre ou plus par mois au cours des 12 derniers mois.

d IMC entre 25,00 et $29,99 \mathrm{~kg} / \mathrm{m}^{2}$.

e IMC supérieur ou égal à $30,0 \mathrm{~kg} / \mathrm{m}^{2}$.

f $3,0 \mathrm{kcal} / \mathrm{kg} /$ jour ou plus.

g 12 fois ou plus par mois

h Trois des choix de réponse concernant l'auto-évaluation de l'état de santé combinés en un seul : excellent, très bon et bon.

facteurs de risque en croissance et pour élaborer des interventions visant cette réduction.

Chez les Premières nations du Nord, la prévalence de l'arthrite, de l'asthme et des maladies cardiaques est plus faible que chez les Autochtones du Sud, celle du diabète est environ la même, et celle de l'hypertension est plus élevée $e^{4}$. Le bilan des facteurs de risque des maladies chroniques est lui aussi variable. Par rapport aux résidents autochtones du Sud, la prévalence de l'embonpoint, de l'obésité et de la consommation régulière
Enfin, la prévalence de l'arthrite, de l'asthme et des maladies cardiaques chez les Métis du Nord canadien est plus faible que celle des Métis du Sud du pays ${ }^{31-33}$. Par rapport aux Autochtones du Sud du Canada, la prévalence de l'embonpoint est semblable, celle du tabagisme quotidien est plus faible, mais celles de l'obésité, de la consommation régulière d'alcool et de la consommation abusive d'alcool sont plus élevées. Comme c'est le cas pour les Inuits et les Premières nations du Nord, le profil des facteurs de risque des Métis est préoccupant en raison de la morbidité associée aux maladies cardiométaboliques, des conséquences sociales et de la mortalité précoce.

Il sera important que les collectivités et les organismes autochtones du Nord travaillent avec les organismes gouvernementaux et les professionnels de la santé pour réduire le profil de risque s'ils espèrent éviter l'épidémie de maladies cardiométaboliques observées chez les Autochtones du Sud du Canada. Or l'environnement nordique est peut-être plus difficile, compte tenu du moins grand nombre de ressources dans les collectivités, du prix élevé des aliments et des effets plus graves du changement climatique ${ }^{34,35}$.

\section{Points forts et limites}

Notre étude comporte des limites. Comme les données de l'ESCC sont fondées sur l'autodéclaration, il se peut que les maladies chroniques et les facteurs de risques comme l'IMC, le tabagisme et la consommation d'alcool aient été sous-estimés. En outre, les seuils d'IMC couramment utilisés pour définir l'obésité et l'embonpoint pourraient ne pas convenir aux populations autochtones ${ }^{36}$. Les répondants pourraient aussi avoir surestimé leur niveau global d'activité physique. Les données sur l'alimentation, bien qu'elles soient liées aux maladies chroniques, n’ont pas pu être incluses, car elles ont été recueillies dans le cadre du module facultatif de l'ESCC. Comme les données de l'ESCC ne concernent que les Autochtones vivant hors réserve, les données des Autochtones qui vivent dans les réserves sont par définition manquantes. Toutefois, les membres des Premières nations, les Inuits et les Métis du Yukon et du 
Nunavut ne vivent pas dans des réserves, ce qui est aussi le cas de plus de $99 \%$ des membres des Premières nations des T.N.-O. ${ }^{5}$. Notre échantillon offre donc une bonne représentation des Autochtones du Nord canadien. Il existe toutefois des limites quant à l'identification des Autochtones dans l'ESCC ${ }^{37}$. Le regroupement des cycles de l'ESCC, en particulier le recours au même échantillon et la dépendance à l'échantillon, constitue aussi une limite. Enfin, le grand nombre de comparaisons peut contribuer à augmenter le risque de résultats significatifs attribuables au hasard.

En dépit de ces limites, cette recherche apporte une contribution importante à la connaissance de la santé des Autochtones du Nord canadien, car ce sont les premiers travaux comparant la prévalence des maladies chroniques et des facteurs de risque chez les membres des Premières nations, chez les Inuits et chez les Métis de cette zone. Nous avons relevé des différences significatives en ce qui a trait aux maladies et aux facteurs de risque entre ces trois groupes autochtones. L'existence de données spécifiques à chaque groupe ethnique est importante pour les organismes politiques autochtones, les décideurs gouvernementaux, les cliniciens et les collectivités, car ces données permettent d'établir des priorités dans les interventions. Certains résultats sont encourageants, mais le profil des facteurs de risque de ces trois populations autochtones du Nord est préoccupant. Le maintien de la surveillance continue des maladies chroniques et des facteurs de risque demeure important pour en suivre les évolutions et pour évaluer l'impact des interventions en santé publique.

\section{Remerciements}

Notre étude a été rendue possible grâce aux Instituts de recherche en santé du Canada. Aucun des auteurs ne présente de conflit d'intérêt.

\section{Références}

1. Statistique Canada. Enquête sur la santé dans les collectivités canadiennes : fichier de microdonnées à grande diffusion, 2005 (cycle 3.1). Ottawa (Ont.) : Statistique Canada; 2006. (n॰82M0013X au catalogue)
2. Bjerregaard P, Young TK, Dewailly E, Ebbesson SOE. Indigenous health in the Arctic : an overview of the circumpolar Inuit population. Scand J Public Health. 2004;32(5):390-395.

3. Waldram JB, Herring A, Young TK. Aboriginal Health in Canada : Historical, Cultural and Epidemiological Perspectives. Toronto (Ont.): University of Toronto Press; 2006.

4. Lix LM, Bruce S, Sarkar J, Young TK. Facteurs de risque et problèmes de santé chroniques chez les Autochtones et les nonAutochtones. Rapports sur la santé. 2009; 20(4):23-32.

5. Northwest Territories Bureau of Statistics. Hay River Reserve-Statistical Profile [Internet]. Yellowknife (NWT) : Northwest Territories Bureau of Statistics; [mis à jour en 2010; consulté le 17 février 2011]. Consultable à partir de la page : http://www .statsnwt.ca/community-data/infrastructure /Hay_River_Reserve.html

6. Statistique Canada. Enquête sur la santé dans les collectivités canadiennes - Composante annuelle (ESCC) : information détaillée pour 2008 [Internet]. Ottawa (Ont.) : Statistique Canada; [modifié le 24 juin 2009; consulté le 26 février 2011]. Consultable à la page : http://www23.statcan.gc.ca/imdb/p2SV_f. pl? Function $=$ getSurvey $\&$ SurvId $=3226 \&$ SurvVer $=1 \&$ InstaId $=15282 \&$ InstaVer $=5 \&$ SDDS $=3226 \&$ lang $=e n \& d b=i m d b \& a d m=8 \&$ dis $=2$

7. Reynolds DL, Chambers LW, DeVillaer MR. Measuring alcohol abuse in the community: consumption, binge-drinking, and alcoholrelated consequences ("alcoholism"). Can J Public Health. 1992;83:441-7.

8. Sarkar J, Lix LM, Bruce S, Young TK. Ethnic and regional differences in prevalence and correlates of chronic diseases and risk factors in northern Canada. Prev Chronic Dis. 2010;7(1):A13.

9. Marmot M, Brunner E. Alcohol and cardiovascular disease: the status of the $U$ shaped curve. BMJ. 1991;303:565-8.

10. Milani RM, Parrott AC, Turner JJ, Fox HC. Gender differences in self-reported anxiety, depression, and somatization among ecstasy/MDMA polydrug users, alcohol/ tobacco users, and nondrug users. Addict Behav. 2004;29:965-971.
11. Longstreth GF, Wolde-Tsadik G. Irritable bowel-type symptoms in HMO examinees. Digest Dis Sci. 1993;38(9):1581-1589.

12. Tverdal A, Thelle D, Stensvold I, Leren P, Bjartveit K. Mortality in relation to smoking history : 13 years' follow-up of 68,000 Norwegian men and women 35-49 years. J Clin Epidemiol. 1993;46(5):475-487.

13. Scott KM, Bruffaerts R, Tsang A et collab. Depression-anxiety relationships with chronic physical conditions : results from the World Mental Health surveys. J Affect Disorders. 2007;103:113-120.

14. Wyatt SB, Winters KP, Dubbert PM. Overweight and obesity : prevalence, consequences, and causes of a growing public health problem. Am J Med Sci. 2006;331(4): 166-74.

15. Statistique Canada. Enquête sur la santé dans les collectivités canadiennes : fichier de microdonnées à grande diffusion 20072008 (cycle 4.1). Ottawa (Ont.) : Statistique Canada; 2009. (n॰82M0013X au catalogue)

16. Santé Canada. Lignes directrices canadiennes pour la classification du poids chez les adultes. Ottawa (Ont.) : Santé Canada, Bureau de la politique et de la promotion de la nutrition; 2003.

17. Rao JN, Wu CF, Yue K. Quelques travaux récents sur les méthodes de rééchantillonnage applicables aux enquêtes complexes. Techniques d'enquête. 1992;18:225-34. (Statistique Canada, n 12001 au catalogue)

18. Bombak AE, Bruce SG. Self-rated health and ethnicity: focus on indigenous populations. Int J Circumpolar Health. 2012;71:18538. DOI : 10.3402/ijch.v71i0.18538.

19. Thomas S, Wannell B. Combiner les cycles de l'Enquête sur la santé dans les collectivités canadiennes. Rapports sur la santé. 2009;20(1):59-65.

20. Rust KF, Rao J. Variance estimation for complex surveys using replication techniques. Stat Methods Med Res. 1996;5:283-310.

21. Yeo D, Mantel H, Liu TP. Bootstrap variance estimation for the national population health survey. Proceedings of the Annual Meeting of the American Statistical Association, Survey Research Methods Section. Baltimore (MD) : American Statistical Association; 1999. 
22. SAS Institute I. SAS/STAT user's guide. Version 9.2. Cary (NC) : SAS Institute; 2009.

23. Young TK, Szathmary EJ, Evers S, Wheatley B. Geographical distribution of diabetes among the native population of Canada: a national survey. Soc Sci Med. 1990;31:129-139.

24. Young TK, Schraer CD, Shubnikoff EV, Szathmary EJ, Nikitin YP. Prevalence of diagnosed diabetes in circumpolar indigenous populations. Int J Epidemiol. 1992;21: 730-736.

25. Sharma S, Hopping BN, Roache C, Sheehy T. Nutrient intakes, major food sources and dietary inadequacies of Inuit adults living in three remote communities in Nunavut, Canada. J Hum Nutr Diet. 2013;26:578-586.

26. Roberts DL, Dive C, Renehan AG. Biological mechanisms linking obesity and cancer risk: new perspectives. Ann Rev Med. 2010;61: 301-316.

27. Hubert HB, Feinleib M, McNamara PM, Castelli WP. Obesity as an independent risk factor for cardiovascular disease: A 26-year follow-up of participants in the Framingham Heart Study. Circulation. 1983;67(5):968977.

28. Rehm J, Mathers C, Popova S, Thavorncharoensap M, Teerawattananon Y, Patra J. Global burden of disease and injury and economic cost attributable to alcohol use and alcohol-use disorders. Lancet. 2009;373:2223-2233.

29. Riva M, Plusquellec P, Juster RP et collab. Household crowding is associated with higher allostatic load among the Inuit. J Epidemiol Commun Health. 2014;68(4):363369. DOI:10.1136/jech-2013-203270.

30. Kral M. "The weight on our shoulders is too much, and we are falling": suicide among Inuit male youth in Nunavut, Canada. Med Anthropol Q. 2013;27(1):63-83.

31. Martens P, Bartlett J, Burland E et collab. Profile of Metis health status and healthcare utilization in Manitoba: a population-based study. Winnipeg (MB) : Manitoba Centre for Health Policy; 2010.
32. Janz T, Seto T, Turner A. Enquête auprès des peuples autochtones de 2006 : un aperçu de la santé de la population métisse [Internet]. Ottawa (Ont.) : Statistique Canada; 2009 [consulté le 9 décembre 2013]. Consultable à la page : http://www.statcan.gc.ca/pub/89 -637-x/89-637-x2009004-fra.htm

33. Bruce SG. Prevalence and determinants of diabetes mellitus among the Metis of western Canada. Am J Hum Biol. 2000;12(4):542551.

34. Wakegijig J, Osborne G, Statham S, Issaluk MD. Collaborating toward improving food security in Nunavut. Int $\mathrm{J}$ Circumpolar Health. 2013;72:21201.

35. Willox AC, Harper SL, Ford JD et collab. Climate change and mental health: an exploratory case study from Rigolet, Nunatsiavut, Canada. Clim Change. 2013; 121:255-270.

36. Lear SA, Humphries KH, Kohli S, Birmingham CL. The use of BMI and waist circumference as surrogates of body fat differs by ethnicity. Obesity. 2007;15(11): 2817-2824.

37. Chan WW, Ng C, Young TK. Nos méthodes d'identification et de recension des Autochtones influent-elles sur l'évaluation du fardeau de la maladie de ce groupe de population?. Maladies chroniques et blessures au Canada. 2013;33(4):312-315. 